\title{
Optical bench development for laser communication OSIRIS mission at Grasse (France) station
}

Duy-Ha Phung, Etienne Samain, Julien Chabé, Clement Courde, Nicolas Maurice, et al.

Duy-Ha Phung, Etienne Samain, Julien Chabé, Clement Courde, Nicolas Maurice, Hervé Mariey, Géraldine Artaud, Aziz Ziad, Christophe Giordano, Eric Aristidi, Dirk Giggenbach, Christian Fuchs, Christian Schmidt, Sabine Klinker, Steffen Gaißer, "Optical bench development for laser communication OSIRIS mission at Grasse (France) station," Proc. SPIE 11852, International Conference on Space Optics - ICSO 2020, 118522B (11 June 2021); doi: $10.1117 / 12.2599371$

SPIE Event: International Conference on Space Optics - ICSO 2021, 2021, Online Only 


\section{International Conference on Space Optics-ICSO 2020}

Virtual Conference

30 March-2 April 2021

Edited by Bruno Cugny, Zoran Sodnik, and Nikos Karafolas
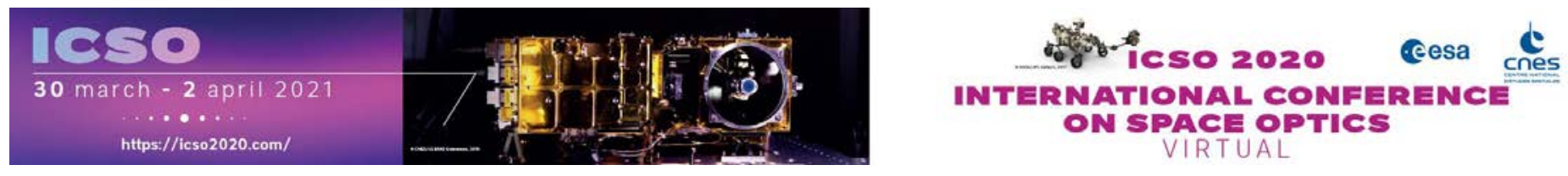

\section{Optical bench development for laser communication OSIRIS mission at Grasse (France) station}

\section{Cesa isoporecestings denes}




\title{
Optical bench development for laser communication OSIRIS mission at Grasse (France) station
}

\author{
${ }^{a *}$ Duy-Ha Phung, ${ }^{b}$ Etienne Samain, ${ }^{a}$ Julien Chabé, a Clément Courde, ${ }^{a}$ Nicolas Maurice, ${ }^{a} H e r v e$ \\ Mariey, ${ }^{\mathrm{c}}$ Géraldine Artaud, ${ }^{\mathrm{d}}$ Azid Ziad, ${ }^{\mathrm{d}}$ Chistophe Giordano, ${ }^{\mathrm{d}}$ Eric Aristidi, ${ }^{\mathrm{e}}$ Dirk Giggenbach, \\ ${ }^{\mathrm{e}}$ Christian Fuchs, ${ }^{\mathrm{e}} \mathrm{Christopher} \mathrm{Schmidt,}{ }^{\mathrm{f}}$ Sabine Klinkner, ${ }^{\mathrm{f}}$ Steffen Gaisser \\ aUniversité Côte d'Azur, Observatoire de la Côte d'Azur, CNRS, IRD, Géoazur, France; \\ ${ }^{\mathrm{b}}$ SigmaWorks, France; ${ }^{\mathrm{c} C N E S}$ - French Space Agency, France; ${ }^{\mathrm{d}}$ Université Côte d'Azur, \\ Observatoire de la Côte d'Azur, CNRS, Lagrange, France; ' Institute for Communications and

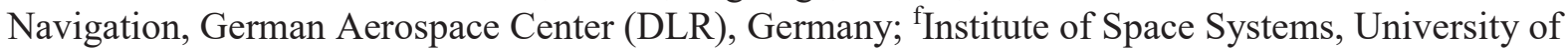 \\ Stuttgart, Germany
}

\begin{abstract}
The Grasse Satellite Laser Ranging (SLR) station (ID7845), part of the International Laser Ranging Service (ILRS) network, participates to various laser applications including SLR, Lunar Laser Ranging (LLR), Time Transfer by Laser Link (T2L2), high-resolution imagery, and debris detection. Since 2014, in collaboration with the France Space Agency (CNES), the Grasse station has been upgrading in order to be able to take part in laser communication (lasercom) experiments. The main challenges of these experiments are the implementation of a complete laser communication chain from space to ground and the characterization of the turbulent atmosphere during the optical data transfer. Several campaigns have been successfully performed and planned since 2015 between Grasse station and lasercom terminal at low Earth orbit, SOTA onboard SOCRATES [5], OPALS integrated on ISS [6], and recently DLR's OSIRIS mission [7]. To prepare for possible higher data-rate lasercom campaigns in the near future (OSIRISv1 at $40 \mathrm{Mbps}$, OSIRISv2 \& Optel $\mu$ at $1 \mathrm{Gbps}$, OSIRISv3 at $10 \mathrm{Gbps}$ ) Grasse SLR station is upgrading on lasercom optical bench, with a fine auto-tracking system and a high sensitivity telecom detector. In the proceeding, we will describe the architecture of our optical bench for high data-rate lasercom link and some primary results of the lasercom link between OSIRISv1 [14] (onboard FlyingLaptop satellite) and the station.
\end{abstract}

Keywords: Grasse Station, Optical Ground Station (OGS), lasercom link, OSIRIS mission, FlyingLaptop, CNES, DLR

*duy-ha.phung@geoazur.unice.fr; phone +33493405421; https://geoazur.oca.eu/fr/acc-geoazur

\section{INTRODUCTION}

In the race of high data rate transmissions for the Space-Ground applications in the future, lasercom is a promising candidate that may replace classical RF technology by its unique features: large bandwidth allowing high data-rate, license free spectrum, less consumption and low mass requirements [1]. Feasibility of lasercom link has been demonstrated at high data-rate through the atmosphere. From first demonstrations at $1.024 \mathrm{Mbps}$ in 1990s [2], the lasercom link has been demonstrated successfully at higher data rate up to 5.625 Gbps bidirectional between the NFIRE satellite and an OGS [3]. Despite of great potential of lasercom, its performance is still limited by the adverse effects of the atmospheric channel (absorption, scattering and turbulence), by the tight link budget related to limited onboard optical power, and by telecom detector sensitivity. These effects may lead to serious degradation in the bit error rate (BER) performance of the lasercom system at high data-rate. However, rapid improvement on optical power for the modulated laser source, pointing and tracking strategy onboard satellite has allowed to integrate lasercom terminal in smaller satellite at low Earth orbit (LEO) for lasercom demonstration.

Since 2014, the SLR Grasse station, based on a 1.54-meter telescope (or MeO telescope), has been upgrading in order to be able to take part in lasercom experiments [4]. Several campaigns have been successfully performed since 2015 between Grasse station and lasercom terminal at low Earth orbit, SOTA (Small Optical Transponder) onboard SOCRATES satellite [5], OPALS (Optical PAyload for lasercom Science) integrated on the International Space Station (ISS) [6]. Atmospheric 
turbulence effects, downlink wavefront, lasercom link performances (pointing, tracking, link budget, bits errors rate) have been analyzed [8], [9]. Since 2017, in order to prepare for future high data-rate lasercom links, the lasercom optical bench of Grasse station has been modified. It has been moved from Nasmyth (mechanically connected to the Alt-Az gimbal of the telescope) to Coudé laboratory (located under the telescope) in order to integrate more auto-tracking system and measurement instruments. In order to establish the lasercom at higher data-rate (Gbps), the ground lasercom detector needs to have a high bandwidth and high sensitivity. Those requirements lead to use a very small detector (diameter of $20-50$ $\mu \mathrm{m})$ that limits the field of view (FoV) of the detector. Particularly, it causes difficulty for maintaining the downlink signal from satellite in the small detector during the satellite pass. With the aim of solving this focalization issue and maintaining the laser spot in small detectors, a fine auto-tracking system, performed by a TipTilt mirror and a camera, is integrated at the input of the optical bench. In addition, the displacement of the optical bench from Nasmyth to Coudé allows creating three sub-apertures, or three independent measurement channels, from $1.54 \mathrm{~m}$ full aperture of the telescope. This subapertures setup compensates the optical loss of the telescope Coudé (after several mirrors and lens) and allows us to achieve a good link budget for each measurement channel. In 2015, the successful link between SOTA and Grasse station has demonstrated the possibility of lasercom when using a small aperture telescope of $40 \mathrm{~cm}$ [8]. A telescope with size of $\sim 50$ $\mathrm{cm}$ and reasonable development cost for development is the tendency for future multi-function telescope. Thus, the experiment setup around $\mathrm{MeO}$ telescope is configured with smaller apertures $(40-50 \mathrm{~cm})$ instead of a full aperture $(1.54$ $\mathrm{cm}$ ) in order to characterize the difficulty and performance of $50 \mathrm{~cm}$ class telescope for high data-rate lasercom link.

Because of strong dependencies of lasercom link performance on weather condition, it is important to measure atmosphere parameters (refraction structure parameter $\mathrm{C}_{\mathrm{n}}{ }^{2}$ profile, Fried parameter, scintillation, wind velocity) during the satellite pass. Recently, the CATS (Calern Atmospheric Turbulence Station) has been installed close to MeO telescope, Figure 1. CATS is composed of a weather station (all sky camera, temperature, humidity, pressure, and wind velocity sensors), Generalized Differential Image Motion Monitor - GDIMM [10] and Moons Limbs Profiler - PBL [11] instruments. Most of the time, a Hufnagel - Valley model of $\mathrm{C}_{\mathrm{n}}^{2}$ profile is used to predict the scintillation and wavefront variance [9] \& [12], [13]. However, to explain the experiment measurement, a scaling factor is necessary. In addition, this scaling factor is not applicable for all observation site, it depends on the local conditions. The lack of $\mathrm{C}_{\mathrm{n}}{ }^{2}$ profile measurement prevents from comparing between the turbulence effects on recorded signals and the theoretical expectation. With the measurements of turbulence parameter from PBL (particularly $\mathrm{C}_{\mathrm{n}}{ }^{2}$ profiles) and GDIMM, one can refine the theoretical model for LEO satellite-to-ground beam propagation through the atmosphere.

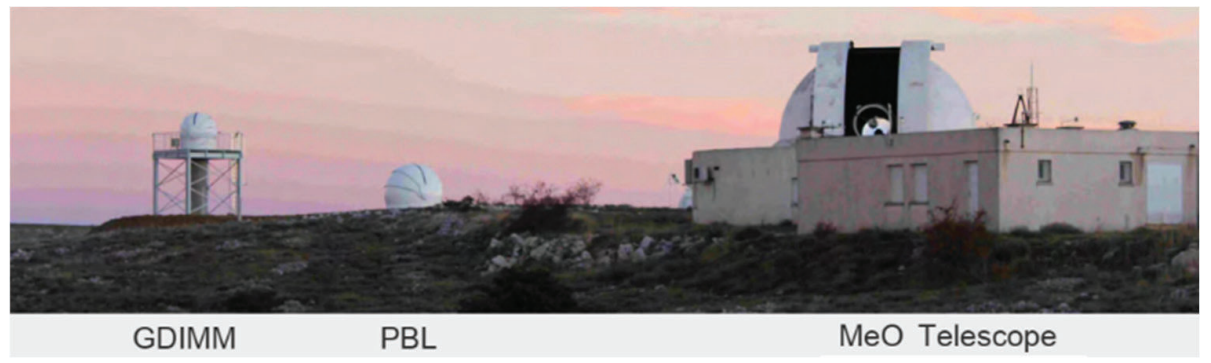

Figure 1. GDIMM \& PBL instruments installed near MeO telescope.

In this proceeding, we present the architecture of Meo telescope and the new optical bench. The design to convert 1.54 full aperture to three sub-apertures in the telescope Coudé will be presented in detail. The auto tracking technique and the components of each measurement channel are also presented. We also provide the first experiment results with different measurements from independent instruments.

\section{OPTICAL BENCH DEVELOPPEMENT}

\subsection{General parameter of MeO telescope}

The $\mathrm{MeO}$ telescope (Metrology and Optics) of Grasse station was designed by the end of the seventies in the framework of a laser-ranging program dedicated to the moon. Since the beginning of 2000, a new organization has been set up permitting to enlarge the initial scientific objectives of the station and to make research and development activities on laser 
links in general. The station is located in the hinterland of Grasse, France at 1270 meters above the sea level. The station is based on a 1.54 meter Ritchey Chretien telescope installed on an Alt-Az mount (Figure 2). The telescope is able to track any targets in the sky at a maximum speed of $5 \%$ s. The mount works with direct drive motors and direct encoders for both azimuth and elevation axes. The pointing accuracy is $<2 \operatorname{arcsec} \mathrm{RMS}$, obtained through a calibration process using absolute position of stars.
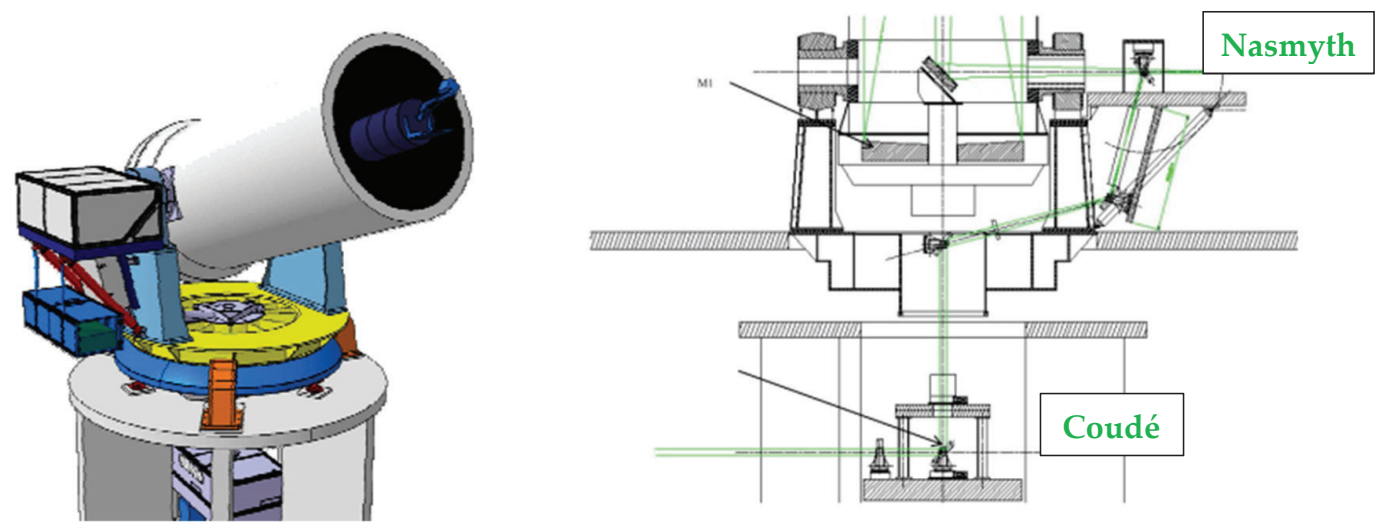

Figure 2. MeO telescope - Overview of the Nasmyth and Coudé path

The instrument has two distinct focus laboratories, Nasmyth and Coudé labs (Figure 2). The Nasmyth lab has a direct access to the primary focus of the telescope, the Coudé lab is connected to the telescope through a coudé path. The optical bench for 2015-2016 lasercom experiment was installed at Nasmyth. All components (coarse tracking camera, telecom detector, and aperture wheel) were mounted in a box of $600 \times 300 \times 250 \mathrm{~mm}^{3}$ [4]. The limitation of the box size and its adding weight connected to the Alt-Az gimbal of the telescope did not allow us to integrate more measurement channels at Nasmyth. Since 2017, we have being developing a new optical bench located in the Coudé of the telescope to prepare future experiments, including links with the OSIRIS's family of optical terminals.
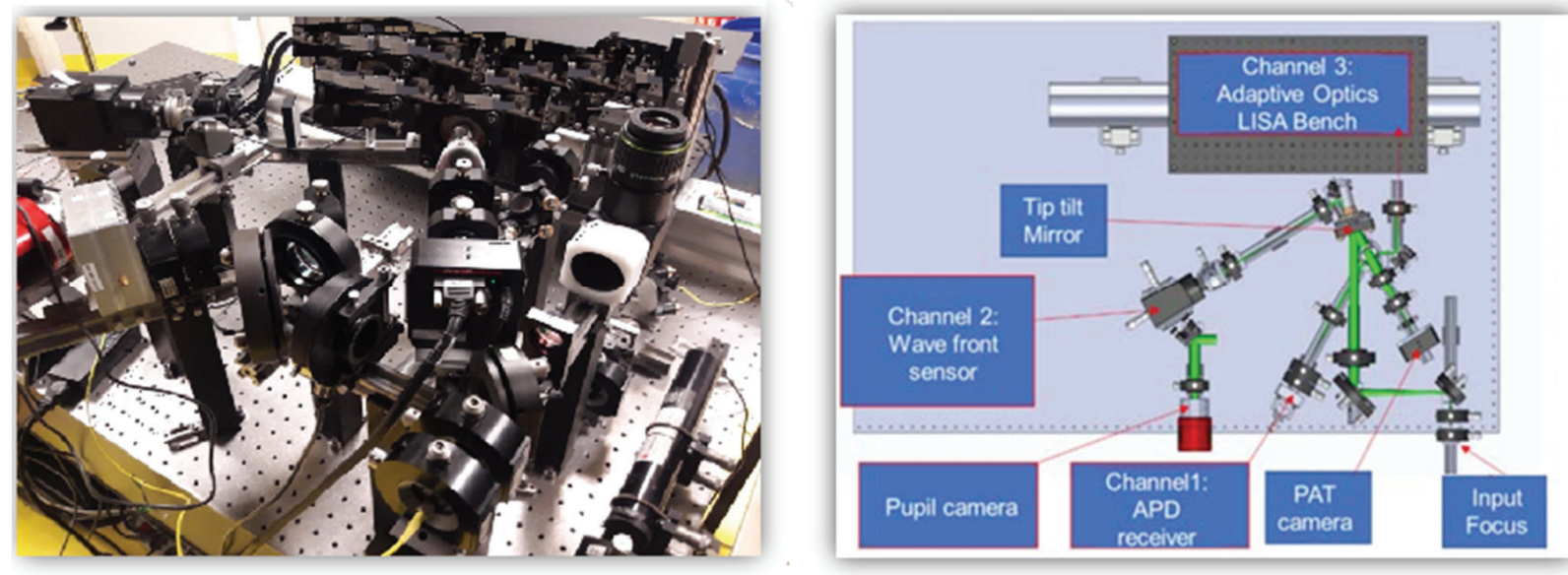

Figure 3. Global view on optical bench at the Coudé of MeO telescope

The incident beam collected by the full aperture of $\mathrm{MeO}$ telescope is coupled to the new optical bench through MeO telescope Coudé. The new bench is a metal table with a dimension of $1800 \times 900 \mathrm{~mm}^{2}$. The different components of each measurement channels are detailed in section $\$ 2.2$. 


\subsection{Architecture of Optical bench for Grasse station - OSIRIS link}

Figure 4 shows the block schema of $\mathrm{MeO}$ telescope with its coudé and new optical bench. The downlink beam from the satellite is collected by $\mathrm{MeO}$ telescope (M1, M2, M3). Then, through MeO telescope coudé M4, M5, D1, M6, the incident beam is coupled to the optical bench via M7 mirror and D2 doublet lens, Figure 4. The beam then goes to the TipTilt mirror, reflected and then split into several parts in order to perform multiple measurements (tracking camera, telecom detector, scintillation measurement, wavefront analyzer and adaptive optics bench - OA LISA). Because of the limitation of link budget (loss in $\mathrm{MeO}$ coudé $\sim 7 \mathrm{~dB}$ ) for multi measurements, three sub-apertures (diameter of $50 \mathrm{~cm}$ ) are created from the full aperture of $\mathrm{MeO}$ telescope (diameter of $1.54 \mathrm{~m}$ ) by using a specific beam splitter system. One maintains the good link budgets for all measurements even when working with smaller aperture. The selection of $50 \mathrm{~cm}$ sub-aperture is to prepare for the development of new generation small telescope with multi-functions.

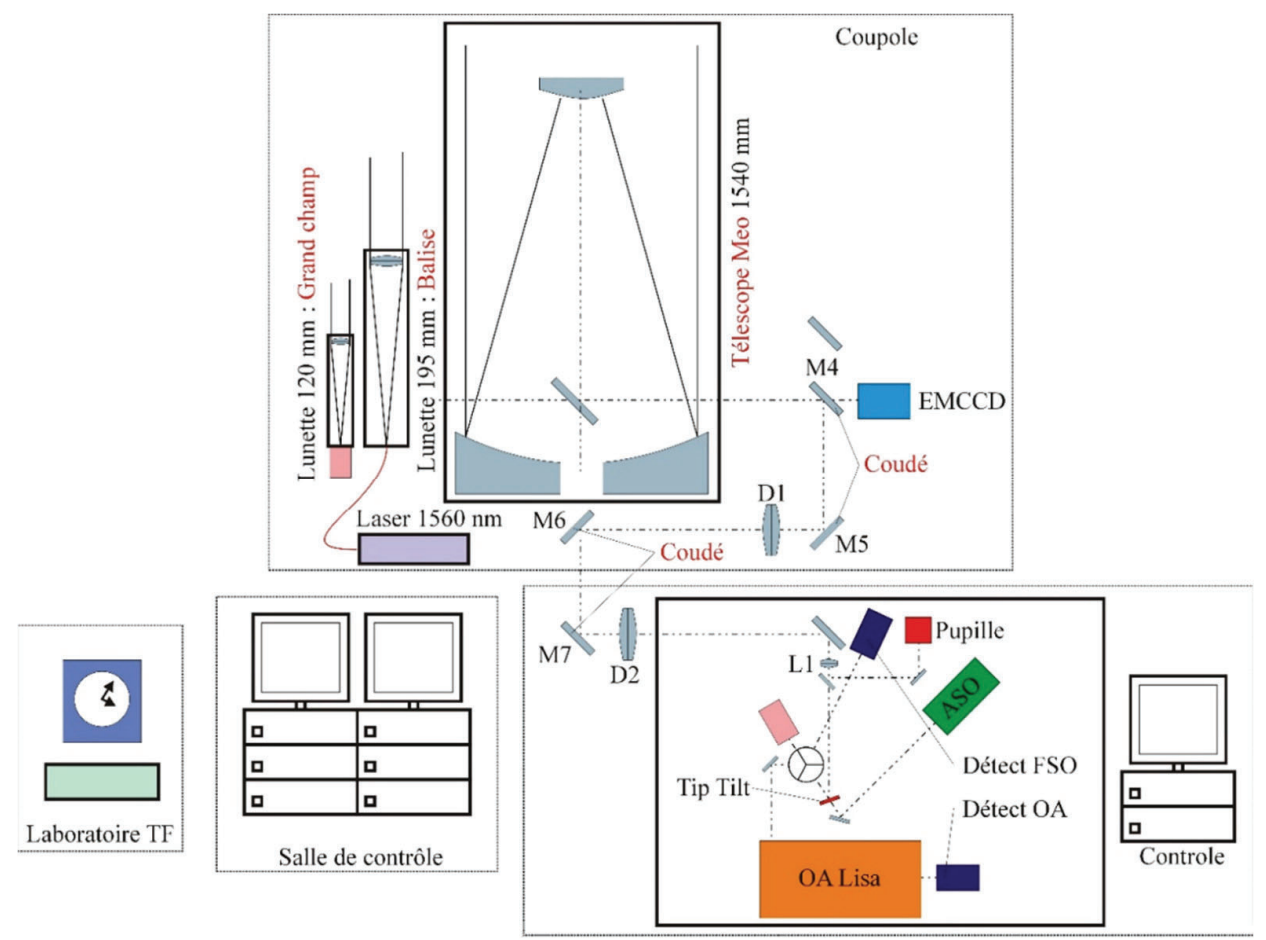

Figure 4. Block Schema of MeO telescope and the new optical bench for OSIRIS - MeO free space link

After coupled to the optical bench, and reflected by TipTilt mirror, the beam goes to the pupil mask with three holes showed in Figure 5-d. The size of holes corresponds to the selected sub-aperture size. Three independents beams (subaperture) are created after the pupil mask. Then, these three beams go to a splitting wedge (90/10) mounted on the same support of the pupil mask, Figure 5-d. 10\% (of each beam) is transmitted and goes to TipTilt camera. 90\% (of each beam) is reflected and go down to the pyramid, show in Figure 5-c. The spot detected by TipTilt camera, composed from three beams (sub-aperture), serves for TipTilt mirror locking-loop. Any misalignment on beam focalization can generate three spots corresponding to three sub-apertures in the TipTilt camera. The $90 \%$ of each beam are then reflected in to three difference directions following three surfaces of the pyramid, showed in Figure 5-d and 5-e. The beam splitting system is performed by an optical prism with three-faces (called the pyramid) at $120^{\circ}$ to each other and $45^{\circ}$ from the base surface. The pupil mask, the splitting wedge and the pyramid are mounted in the same rotation support, showed in Figure 5-d and 5-e. After the pyramid, each of three beams goes to three different directions following the reflecting surfaces of the pyramid, three outputs shown in Figure 5-d. The first beam is dedicated for telecom signal detection (by a lab-developed high-sensitivity photodetector, discussed in section §2.4). The second part is dedicated for a Shack Hartmann wavefront sensor (SHWFS) to characterize atmospheric turbulence effect during experiment. The other is dedicated to OA LISA 
bench, this bench is developed and operated by ONERA - French Aerospace Lab, for fiber coupling test using high-speed adaptive optics.
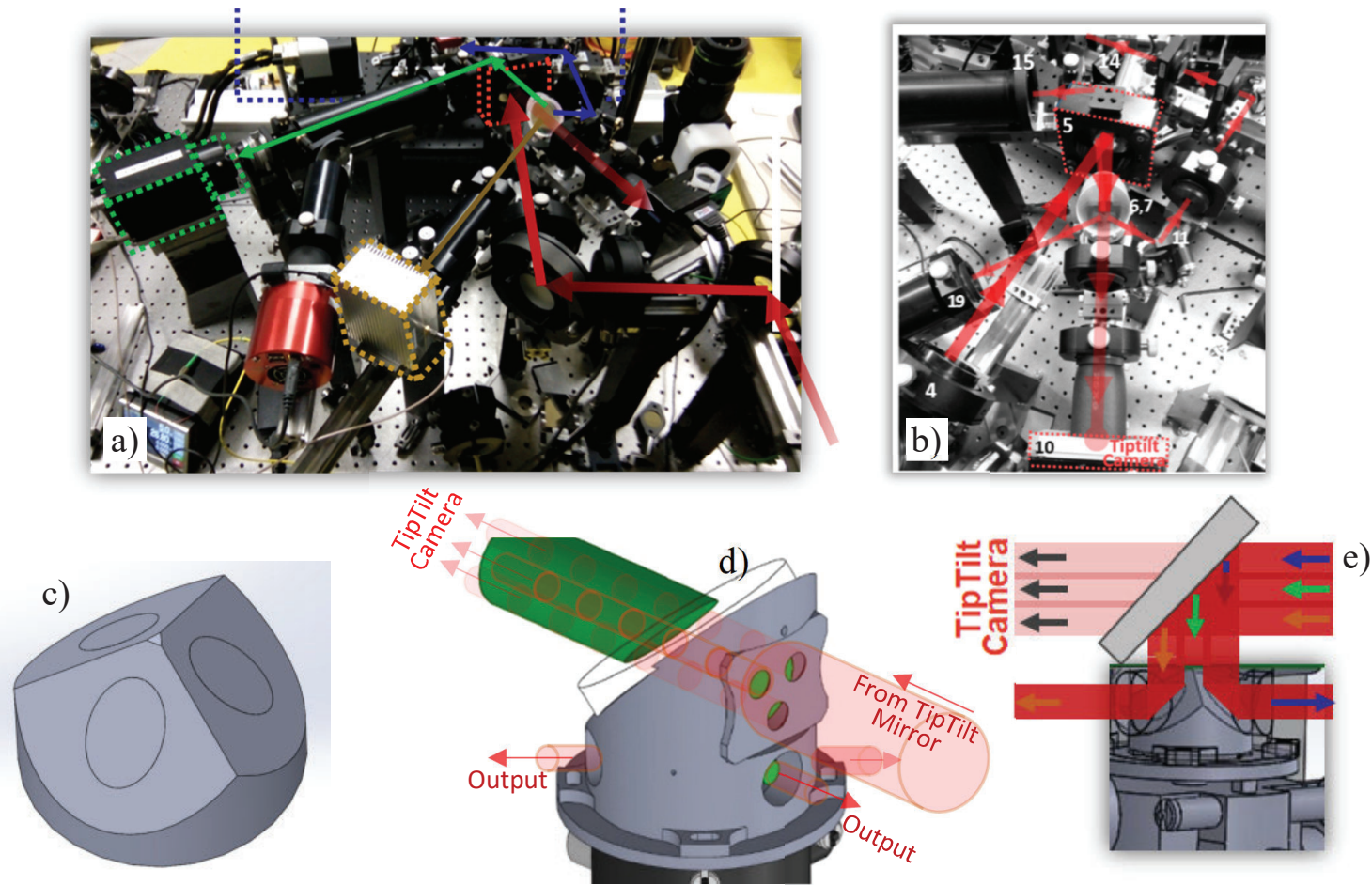

Figure 5. Global view on the optical bench (a); Zoom on the pyramid and TipTilt system (b)

Pyramid splitter (c) with 3 holes mask in the mount (d) and sub-aperture beams direction (e)

The cameras (TipTilt camera, SHWFS camera) and the TipTilt mirror controller are monitored by computer mounted near the bench. This computer is connected to the control center of the telescope in order to perform auto-tracking during the satellite pass. The TipTilt mirror system serves for fine tracking with a dynamic of \pm 30 arcsec.

The coarse tracking is performed by a wide field camera and a $200 \mathrm{~mm}$ telescope mounted on $\mathrm{MeO}$ telescope (Figure 6). The $200 \mathrm{~mm}$ telescope is constituted by a divergence lens and apochromatic doublet lens (diameter of $195 \mathrm{~mm}$, focal length of $1755 \mathrm{~mm}$ ) installed inside a carbon tube. The very large FoV of $2200 \times 1760 \operatorname{arcsec}^{2}$ and an aperture of $200 \mathrm{~mm}$ allow us to have a performant system for satellite finding and coarse tracking.

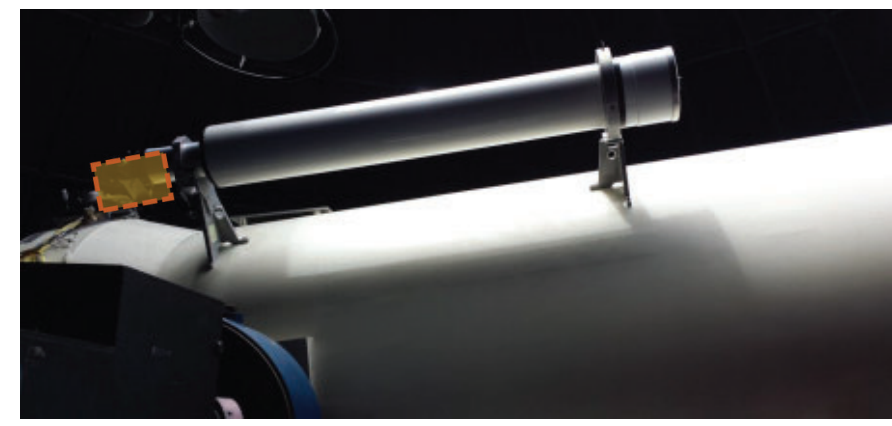

Figure 6. Wide filed Camera (1760x2200 $\left.\operatorname{arcsec}^{2} \mathrm{FoV}\right)$ installed on $200 \mathrm{~mm}$ telescope mounted on MeO telescope.

This $200 \mathrm{~mm}$ telescope can serve also for uplink beam transmitter with a high power laser source in the case where the pointing onboard satellite needs an uplink beam to establish the lasercom link (case of SOTA, OPALS and OSIRISv2). 
The divergence of the uplink beam can be controlled by software allowing for an adjustment of the uplink optical power density in the real time. In this case, the wide field camera is mounted on another refractive telescope (also mechanically connected to $\mathrm{MeO}$ telescope tube, but smaller).

\subsection{Pointing and auto-tracking}

When the downlink beam from satellite is detected in the wide field camera, the autotracking of MeO telescope is activated. The coarse tracking is performed by sending directly correction signal to $\mathrm{MeO}$ telescope. The correction speed of the coarse tracking is decided by the controller of $\mathrm{MeO}$ telescope, and set to $10 \mathrm{~Hz}$. The coarse tracking brings the downlink beam into TipTilt camera FoV in order to enable the fine tracking. When the laser spot is detected by the TipTilt camera, the fine tracking is activated. Its maximum bandwidth of correction is $100 \mathrm{~Hz}$ limited by the maximum frame rate of the TipTilt camera (Hamamatsu C11512-02). The dynamic of TipTilt mirror is \pm 30 arcsec. When TipTilt mirror correction approaches its limit, the coarse tracking sends corresponding correction (calculated from TipTilt mirror correction signal) to the controller of $\mathrm{MeO}$ telescope. By changing the set point of coarse tracking on wide field camera one discharges the TipTilt mirror correction, and sets it to zero. The downlink beam from OSIRISv1 satellite detected by wide field camera and by TipTilt camera is shown in Figure 7 and Figure 8, respectively.

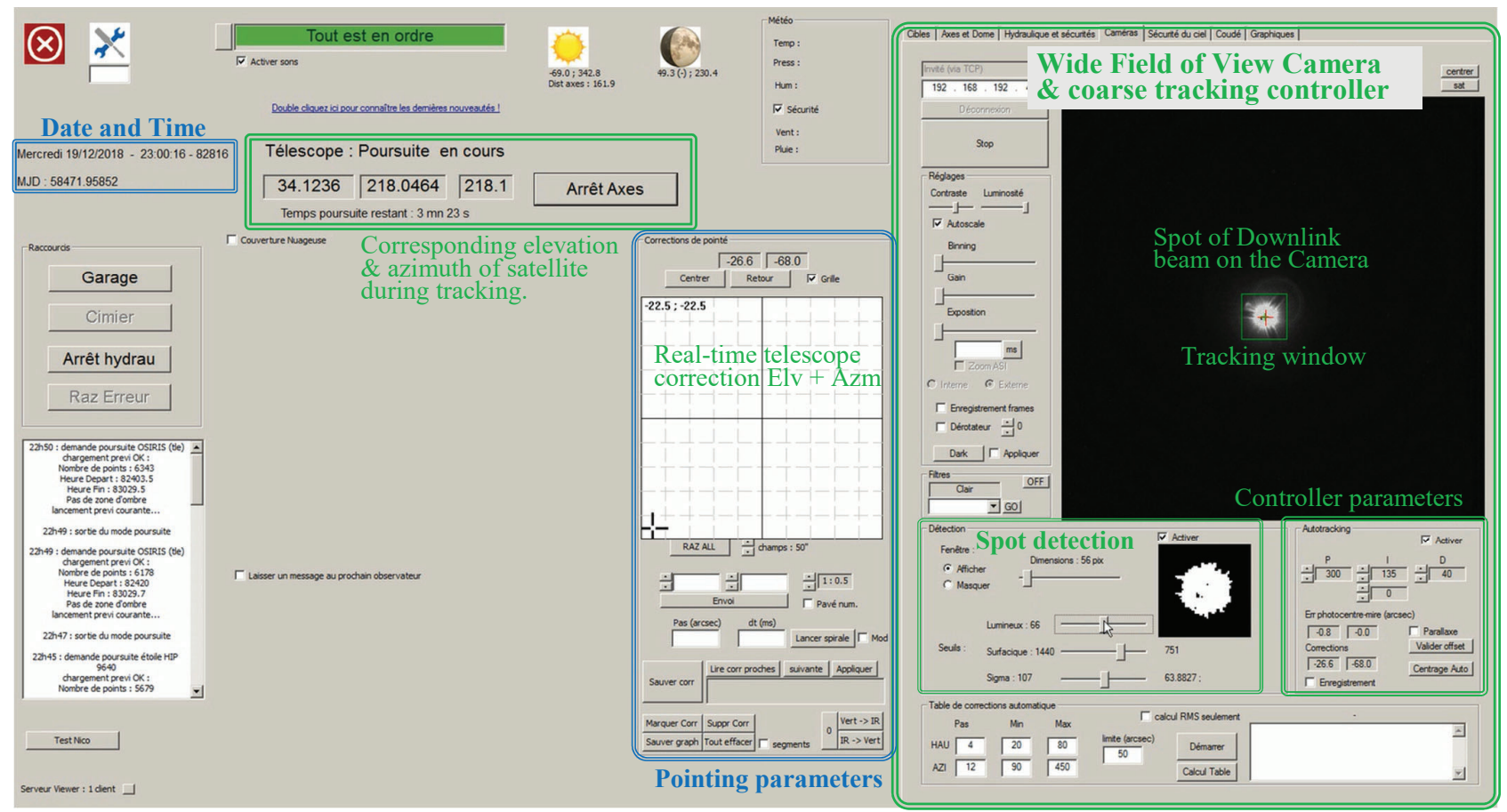

Figure 7. Screen shot of Satellite coarse-tracking controller software and Wide Field camera

In case where the TipTilt camera detects the downlink beam spot and the TipTilt mirror is activated, the coarse tracking by wide field camera is maintained active. Its set point will be only varied when the TipTilt mirror correction approaches the limit of $15 \mathrm{arcsec}$. The TipTilt discharging method is to solve the misalignment issue between the zero center of wide field camera (using $200 \mathrm{~mm}$ telescope) and the optical bench (at the Coudé of $\mathrm{MeO}$ telescope) during the satellite tracking. 


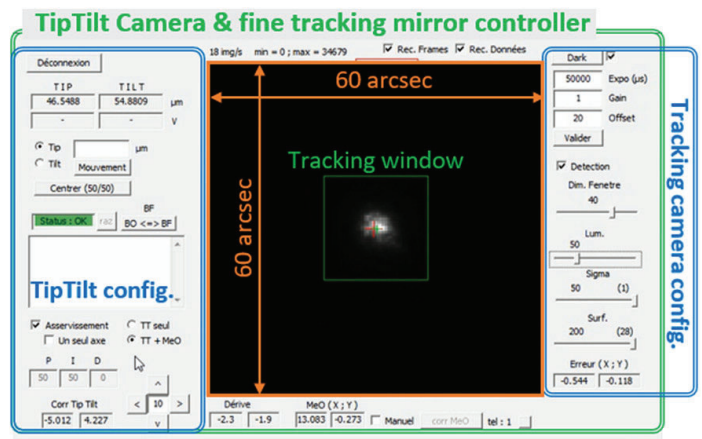

Figure 8. Screen shot of the fine tracking controller software by using TipTilt Camera + Mirror

When TipTilt mirror is activated, the downlink beam spot is locked on the center of TipTilt camera with an error of 1 arcsec RMS at short time range $(20 \mathrm{~ms})$. In addition, it maintains the spot of the downlink beam in the small detection zone of the telecom detector during the whole satellite pass with a stability $<0.1 \operatorname{arcsec}$ at $2000 \mathrm{~s}$.

\subsection{Telecom signal detection}

For LEO direct to Earth demonstrations, we have developed three high sensitivity telecom detectors corresponding to three different data-rates: $40 \mathrm{Mbps}$ (usable with OSIRISv1), $1.0 \mathrm{Gbps}$ and $10 \mathrm{Gbps}$. Figure 9 shows the telecom detectors designed and integrated by SigmaWorks: STH301-000-005 (bandwidth of 26 MHz) for $40 \mathrm{Mbps}$ detection and STH201101-002 (bandwidth of 0.85 GHz) for $1.0 \mathrm{Gbps}$ detection. The detector STH201-010-003 (bandwidth of $6 \mathrm{GHz}$ ) for 10 Gbps detection is under development.
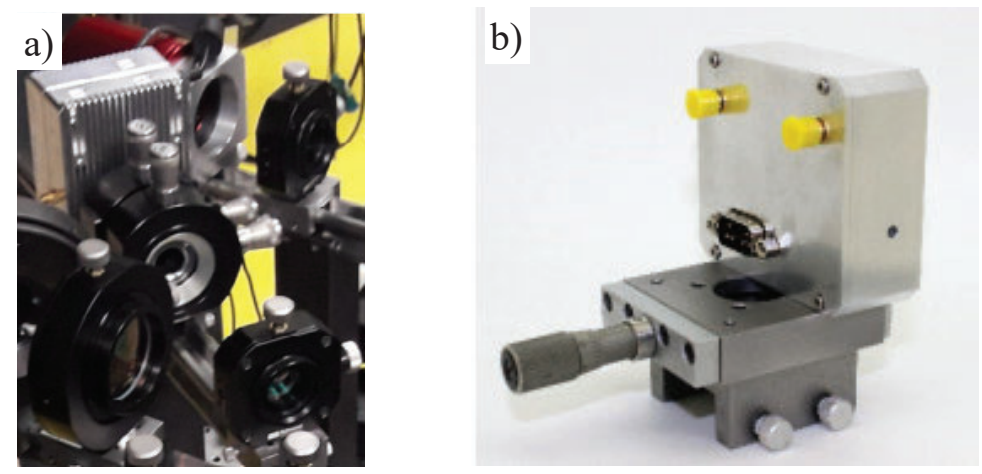

Figure 9 . Telecom detector on the bench, STH301-000-005 at $26 \mathrm{MHz}$ (a) and STH201-101-002 at 0.85 GHz (b)

The BER detection performance (at $10^{-10}$ ) of the detector are respectively $-48 \mathrm{dBm}$ and $-38 \mathrm{dBm}$.

The output of the telecom detector is connected to a high-speed digitizer CSE123G2-GS from GAGE. The digitization speed is up to $6.125 \mathrm{GSps}$ in continuous mode with 16 bits resolution. All signals acquired during a given pass are synchronized together through a Network Time Protocol (NTP) server that is connected to a Hydrogen Maser. The Hydrogen Maser and the NTP server is located at $70 \mathrm{~m}$ from the telescope in the time/frequency laboratory of the station.

The optical power level of the received signal (DC component) is measured also by the telecom detector. The digitalized signal is downsampled by software to $2 \mathrm{kSps}$ for flux fluctuation measurement. The noise of the scintillation measurement chain (at $2 \mathrm{kSps}$ ) is roughly $1.8 \mathrm{pW}$ RMS, which is acceptable with regards to the dynamics of the measured optical power level (from $-70 \mathrm{dBm}$ or $0.1 \mathrm{nW}$ to $-40 \mathrm{dBm}$ or $100 \mathrm{nW}$ ). 


\subsection{PBL and GDIMM}

PBL (Moon Limbs Profiler) uses the image motions of the Moon limbs at different positions to estimate $\mathrm{C}_{\mathrm{n}}^{2}$ for 24 layers at different altitudes from $50 \mathrm{~m}$ to $24 \mathrm{~km}$ [11]. The Moon was at elevation of $50 \mathrm{deg}$ during OSIRISv1 passes on Dec.19, 20 , and closer to the descending part of OSIRISv1's passes. The $\mathrm{C}_{\mathrm{n}}{ }^{2}$ profiles measured by PBL are shown in Figure 10 at 24 layer of altitude from $50 \mathrm{~m}$ to $24 \mathrm{~km}$. The measurement rate is about of one measure for every 150 seconds. From these measured profiles, one can estimate the scintillation index, the Fried parameter and compare them with the direct measurements from lasercom link. Figure 10 shows the time series of $\mathrm{C}_{\mathrm{n}}{ }^{2}$ recorded at Grasse station during nighttime and daytime when the sky was clear. $\mathrm{C}_{\mathrm{n}}{ }^{2}$ depends on turbulence conditions that can vary extensively within a 2 hours time scale, or day after day. Thus, using a unique $\mathrm{C}_{\mathrm{n}}^{2}$ profile for an observation site at all times is not recommended.
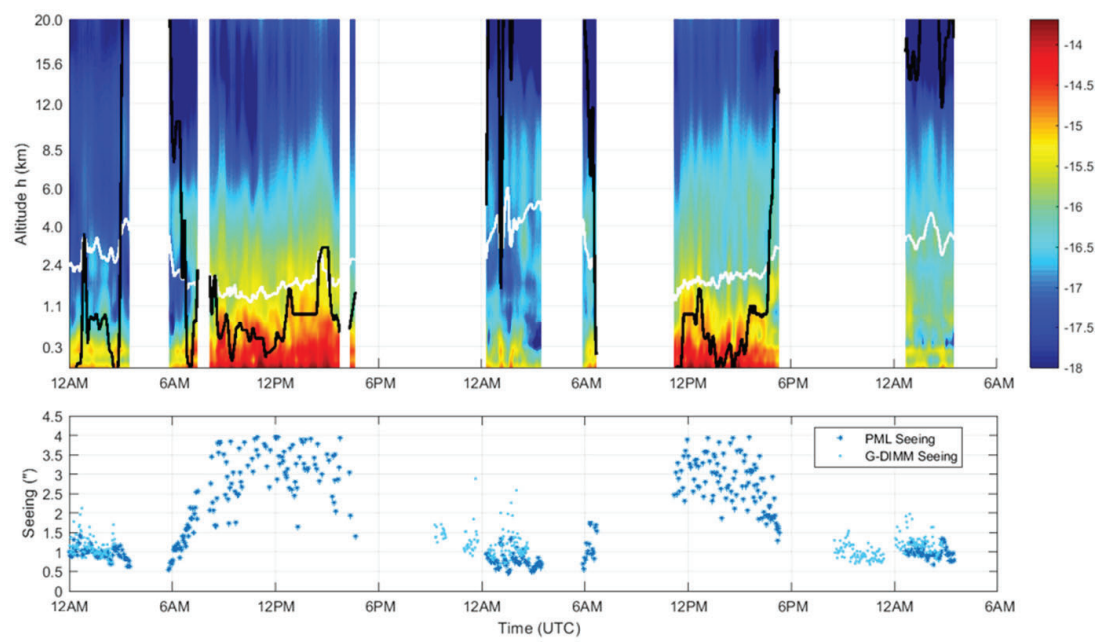

Figure 10. Example of $\mathrm{C}_{\mathrm{n}}{ }^{2}$ (h) profiles acquired from June 20-June 22, 2019 as a function of time [11] (square root scale for altitude $\mathrm{h}$ ) and corresponding seeing parameter measured from PBL (PML seeing) and GDIMM

GDIMM (Generalized Differential Image Motion Monitor) uses stars for the image motion measurement during nighttime. It is based on a small telescope equipped with a 3-holes mask at its entrance pupil [10] in order to measure turbulence parameters: angle-of-arrival variance or Fried parameter $r_{0}$, scintillation index and particularly optical coherence outer scale $L_{0}$, Figure 11 shows an example of GDIMM output during the night of Dec 20, 2018. All parameters measured by GDIMM are computed to a zenith angle of 0 deg and for the wavelength 500nm. The GDIMM measures the seeing and other atmosphere parameters every 90 seconds.
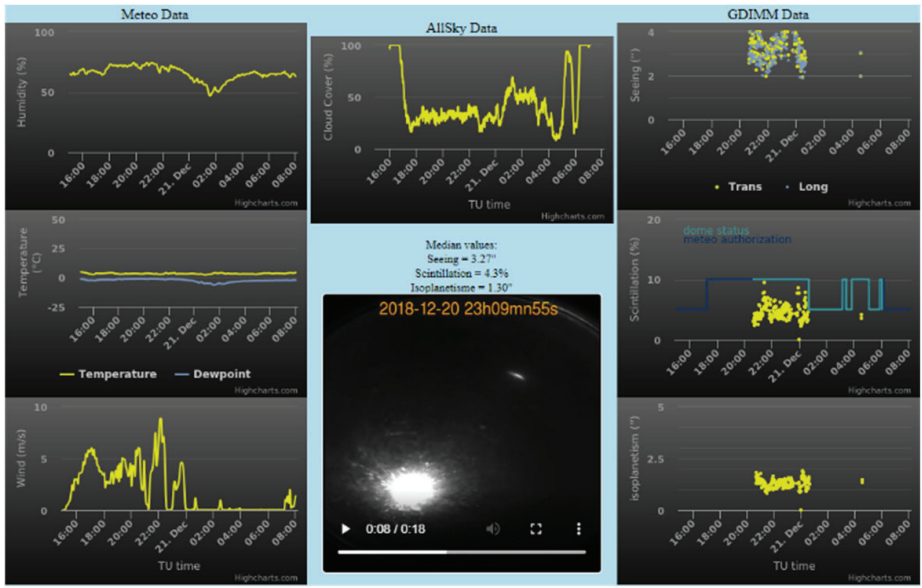

Figure 11. Example of GDIMM measurement - Dec.20.2018: with humidity, temperature, wind velocity, cloudy cover rate, all sky camera, seeing parameter, scintillation and isoplanatic angle. 
During lasercom experiment, for the first time, one has three independent instruments that observe the atmospheric turbulence effect on three independent sources: MeO optical bench observes the lasercom downlink beam of OSIRISv1 satellite using a Shack Hartmann sensor; PBL observes the Moon limbs; GDIMM observes the stars. The comparison between experiment data recorded of these instruments allows us to understand the turbulence effects on lasercom downlink beam. The results of this analysis will be published in an upcoming article.

\section{VALIDATION OF THE GRASSE STATION SET-UP WITH OSIRISV1}

\subsection{Description of the links}

Thanks to German Aerospace Center (DLR), three passes of OSIRISv1 [14] on Dec.18, 19, 20-2018 has been proposed for Grasse station in order to validate the instrumental set-up. Orbit info (Two Line Element - TLE file) and laser ON/OFF period of each satellite pass has been communicated by DLR $3-5$ hours before the satellite pass. During these successful passes, the sky was clear with strong wind on Dec.19 and $20-2018$, and the sky was cloudy (no star was visible on eyes) for the first test on Dec.18-2018. Figure 12 shows elevation, azimuth angles of the satellite and Laser ON/OFF periods (by orange part on elevation curves) for three proposed passes.
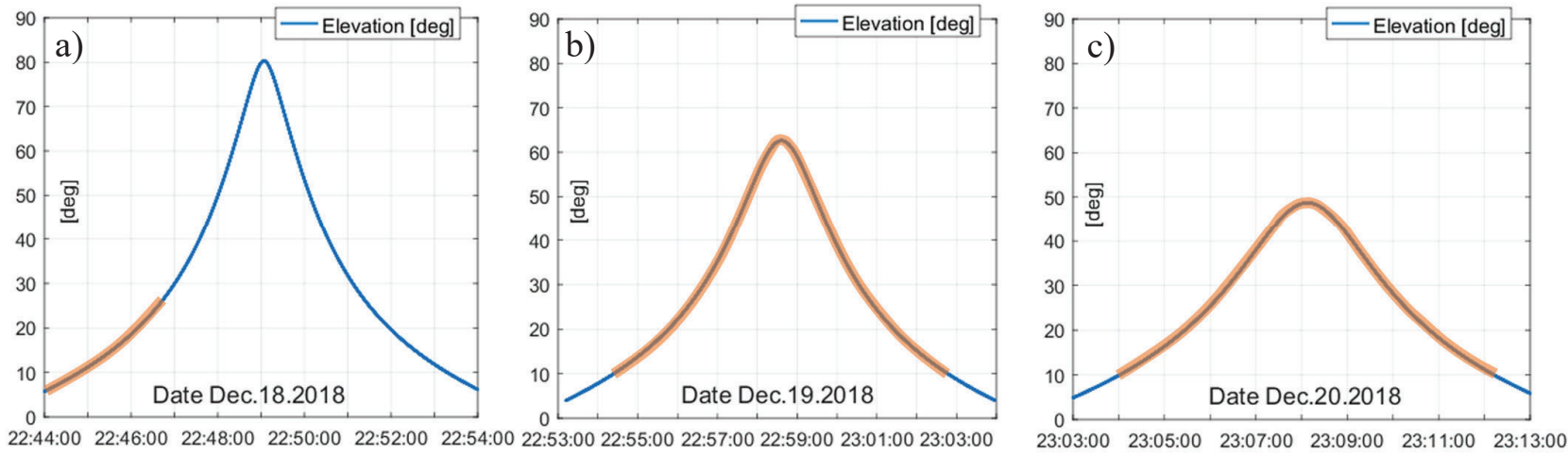

Figure 12. Elevations and corresponding ON/OFF onboard laser during three passes Dec.18 (a),19(b),20(c).2018

Laser ON/OFF periods are illustrated by orange part on elevation curves.

The culminations of three passes Dec. $18,19,20$ are $80^{\circ}, 63^{\circ}$ and $49^{\circ}$, respectively

Table 1 shows some weather information of three passes. The seeing parameter of Dec 18 was not available because the sky was cloudy.

Table 1. Weather condition during three OSIRISv1 passes on Dec.18 22h45, Dec.19 22h59, Dec.20 $23 \mathrm{~h} 08$.

\begin{tabular}{|c|c|c|c|c|}
\hline Parameters & & Dec 18 & Dec 19 & $\overline{D \text { Dec } 20}$ \\
\hline Visibility & & Cloudy & Clear & Clear \\
\hline Temperature $\quad\left[{ }^{\circ} \mathrm{C}\right]$ & & 0.3 & 0.5 & -0.4 \\
\hline Humidity $[\%]$ & & 84 & 92 & 86 \\
\hline Pressure $\quad[\mathrm{hPa}]$ & & 879 & 873 & 872 \\
\hline Wind speed $\quad[\mathrm{km} / \mathrm{h}]$ & & 7.8 & 12.4 & 15.1 \\
\hline Wind direction $\quad[\mathrm{deg}]$ & & 255 & 225 & 315 \\
\hline Seeing at $500 \mathrm{~nm} \&$ zenith & {$[\operatorname{arcsec}] *$} & NA & 3.2 & 3.9 \\
\hline
\end{tabular}

\subsection{Auto-tracking system results}

The Figure 13 below shows all FoV sizes (in the same range) corresponding to 4 detectors ( 3 cameras and 1 photodiode) used in the experiment. When TipTilt mirror is activated, the downlink beam spot is locked on the center of TipTilt camera with an error of 1 arcsec RMS at short time range $(50 \mathrm{~Hz})$. The spot size of downlink beam at telecom detector is $5 \times 5$ 
$\operatorname{arcsec}^{2}$ (FWHM) that is fully covered by its FoV of 30 arcsec. Thus, the telecom detector can measure correctly the downlink signal during the satellite pass.
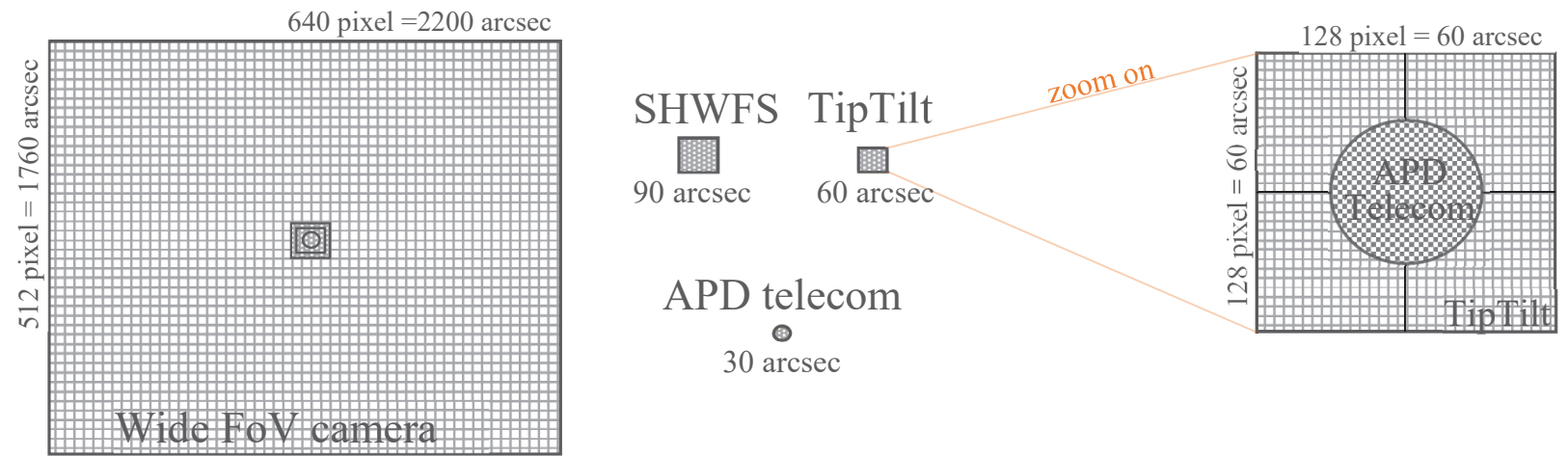

Figure. 13. Field of view of different detectors used in the experiment.

The ratios of detector real sizes are respected in this illustration.

From TLE file sent by DLR, we generate orbit prediction at time scale of $100 \mathrm{~ms}$. Figure 14 shows the orbit prediction (elevation, azimuth, corresponding elevation and azimuth velocity) for the pass on Dec.20. Weather condition for this pass: The sky was clear with strong wind. Strong turbulence condition: around of 3.9 arcsec measured at 23:10:00 by GDIMM.
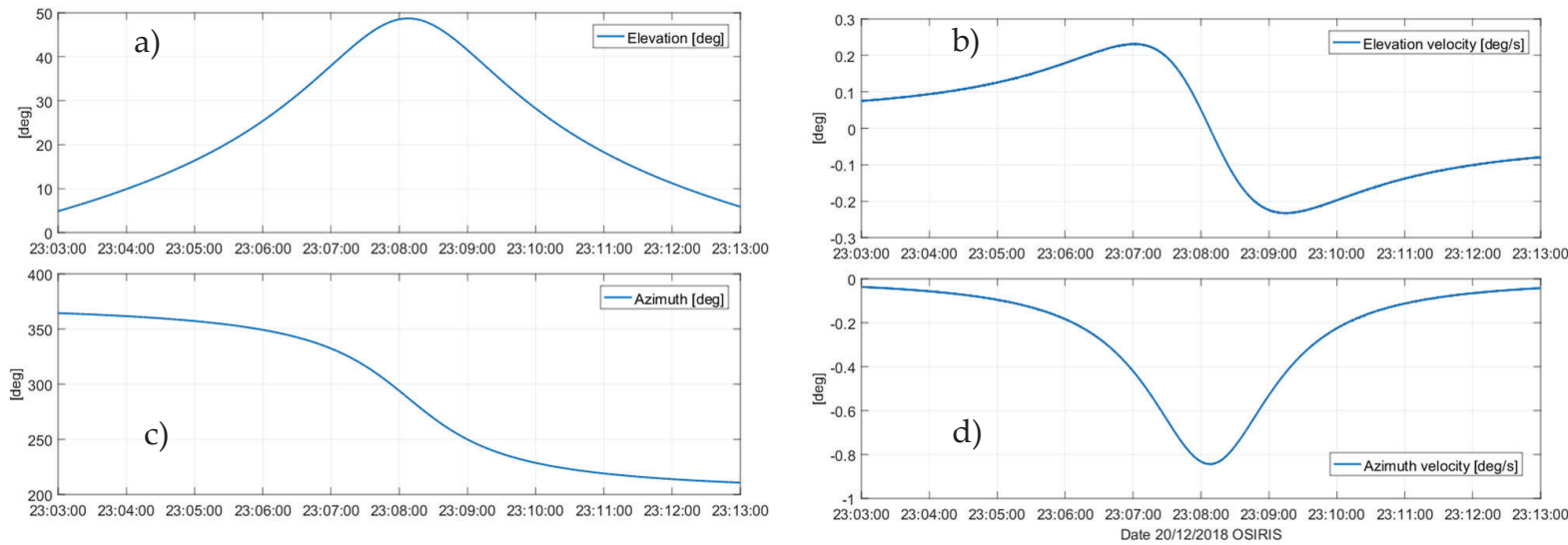

Figure. 14. Orbit information of OSIRISv1 (view from MeO OGS using TLE) - Dec.20.2018 satellite pass Left side: Satellite Elevation (a), Azimuth (b): Elevation velocity (c) and Azimuth velocity (d).

The downlink beam was detected at OGS in the wide field camera with a prediction error $<20$ arcsec when the satellite is at low elevation (10 deg). Coarse tracking is firstly activated by sending directly the correction calculated from wide field camera detection to the telescope controller. Then, fine tracking is activated; TipTilt mirror is locked on spot position detected by TipTilt camera. The auto-tracking was activated in most of the time during satellite pass, more than 7 minutes. The coarse and fine trackings maintain the downlink beam coupled in all detectors in spite of satellite prediction error.

The prediction error of OSIRISv1 (from TLE) is estimated to be roughly 100 arcsec pic-to-pic in elevation and 220 arcsec pic-to-pic in azimuth, Figure 15-a. Small variation in coarse corrections, Figure 15-a, relates to a discharge of TipTilt controller when TipTilt correction approaches the limit. Although large fluctuation of downlink optical power, coarse and fine tracking were always activated during the pass until OSIRISv1 laser was turned off. The downlink beam is stabilized in all detectors with an RMS error $<1$ arcsec during the satellite pass, Figure 15-c. 

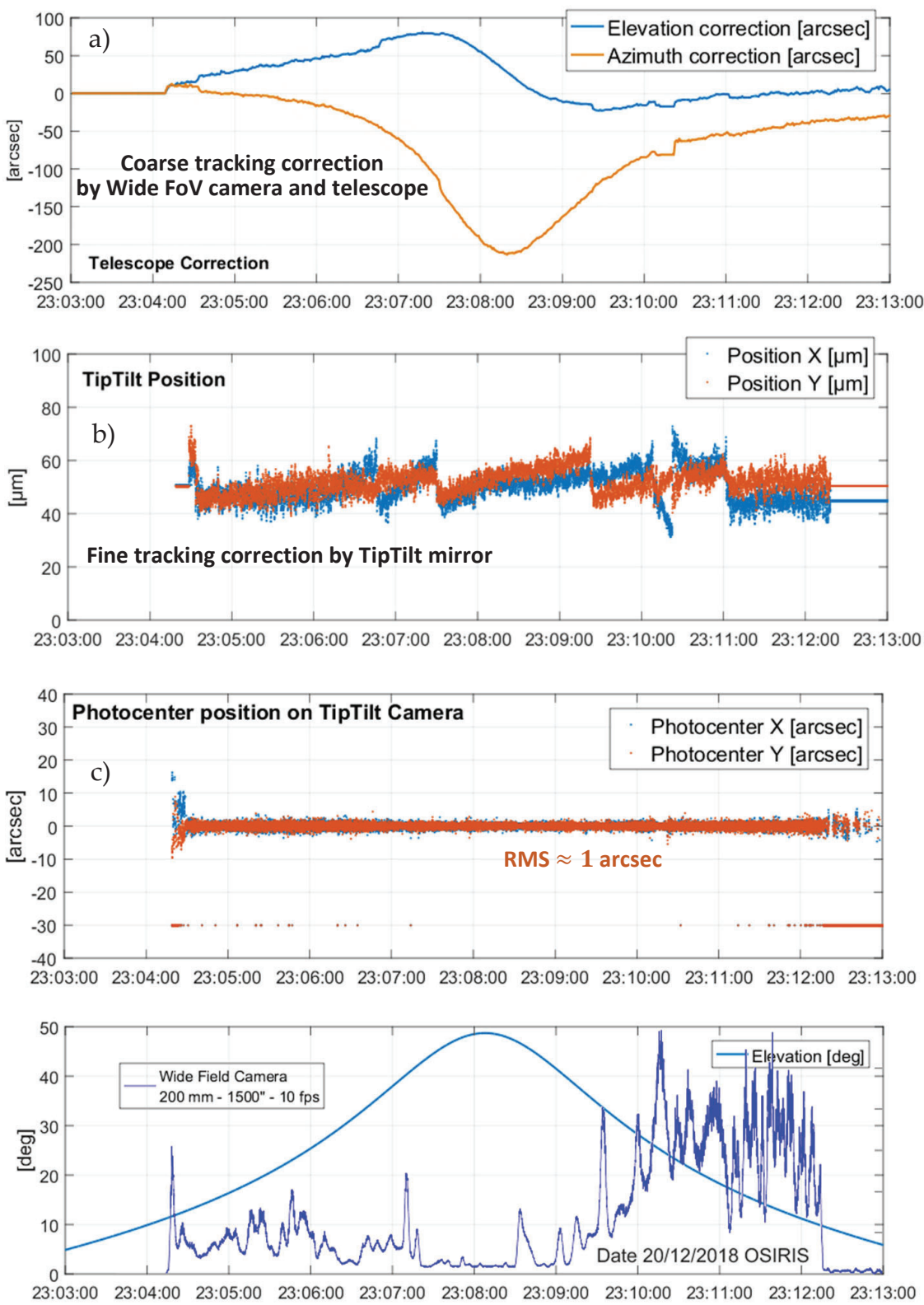

Figure. 15. Coarse and Fine auto-tracking during OSIRISv1 pass

(a) Coarse tracking correction by Wide FoV camera and telescope; (b) Fine tracking correction by TipTilt mirror; (c) Laser spot position detected by TipTilt camera, RMS $<1$ arcsec; (d) Optical power detected by Wide FoV camera.

The first downlink signal of OSIRISv1 has been detected by MeO telescope on the Dec.18, during 90 seconds when the satellite elevation was from $7^{\circ}$ to $20^{\circ}$. A pointing bias (on the ascending part) was observed. This bias has been since reduced by optimization of the OSIRISv1 system on Flying Laptop. The measured received powers were common in all detectors (wide field camera, TipTilt camera, Shack Hartmann wavefront sensor and telecom photodetector). The fluctuations at $0.1-0.3 \mathrm{~Hz}$ observed in downlink signal relate to satellite bias pointing. 
The link budget for $50 \mathrm{~cm}$ aperture receiver was good enough for the telecom detector performance to detect the data-bits from the telecom signal. The data analysis on flux fluctuation, link performance, Bit error rate, and the comparison with PBL and GDIMM measurements will be presented in near future publication.

\section{CONCLUSION AND PERSPECTS}

The Grasse station was upgraded with a new optical bench at the Coudé of the telescope. This upgrade allows to integration a fine-tracking system (based on a TipTilt mirror) to the auto-tracking of the $1.54 \mathrm{~m}$ telescope. The displacement of the optical bench from Nasmyth to a $1800 \times 900 \mathrm{~mm}^{2}$ table at the Coudé of the telescope facilitate us to integrate more measurement instrument in the optical bench. We have presented the splitting technique of the new optical bench that generates 3 independent sub-apertures from the full aperture of the telescope. This pyramid and wedge configuration allow us to achieve three different measurement channels but still keeping a good link budget for each measurement. On December 18, 19, 20 2018, three successful links between Grasse station and the satellite OSIRISv1 have been established. This is the first time a downlink beam from a body-pointing lasercom satellite has been detected in Grasse station. The combination between coarse and fine tracking allows the auto-tracking system maintain the downlink laser from satellite to the small detection zone of the telecom detector during the whole satellite pass. The detail analysis (downlink flux level, flux fluctuation, BER performance and comparison to the theoretical expectation) on the collected data during three successful passes will be published in the near future.

\section{REFERENCES}

[1] Vincent W. S. Chan, "Free-Space Optical Communications", Journal of Lightwave Technology Vol. 24, Issue 12, pp. 4750-4762 (2006)

[2] K. E. Wilson, M. Jcganathan, J. James, G. Xu and J. R. Lesh, "Results FromPhase- 1 and Phase-2 GOLD Experiments" JPL TDA Progress Report 42-127, (1996).

[3] R. Fields et al., "5.625 Gbps bidirectional laser communications measurements between the NFIRE satellite and an Optical Ground Station," 2011 International Conference on Space Optical Systems and Applications (ICSOS), Santa Monica, CA, 2011, pp. 44-53, (2011).

[4] E. Samain, N. Maurice, D-H. Phung, et al, "First Free Space Optical Communication in Europe Between SOTA and MeO Optical Ground Station", Proceedings of ICSOS2015 26-28 Oct. 2015, (2015).

[5] Alberto Carrasco-Casado, Hideki Takenaka et al, "LEO-to-ground optical communications using SOTA (Small Optical TrAnsponder) - Payload verification results and experiments on space quantum communications", Acta Astronautica, Volume 139, Pages 377-384, (2017)

[6] B. V. Oaida, M. J. Abrahamson, R. J. Witoff, J. N. Bowles Martinez and D. A. Zayas, "OPALS: An optical communications technology demonstration from the International Space Station," 2013 IEEE Aerospace Conference, Big Sky, MT, 2013, pp. 1-20, (2013).

[7] Christian Fuchs, Christopher Schmidt, "Update on DLR's OSIRIS program," Proc. SPIE 11180, International Conference on Space Optics - ICSO 2018, 111800I, (2019).

[8] D-H. Phung, E. Samain, N. Maurice et al, Telecom and scintillation first data analysis for DOMINO: laser communication between SOTA, onboard SOCRATES satellite, and MEO optical ground station, and MEO OGS, Proceedings Volume 9739 SPIE LASE, (2016), https://doi.org/10.1117/12.2218524

[9] Cyril Petit, Nicolas Védrenne, Marie Therese Velluet, Vincent Michau, Geraldine Artaud, Etienne Samain, Morio Toyoshima, "Investigation on adaptive optics performance from propagation channel characterization with the small optical transponder," Opt. Eng. 55(11), 111611 (2016),

[10] E Aristidi, A Ziad, J Chabé, Y Fantéi-Caujolle, C Renaud, C Giordano, "A generalized differential image motion monitor", Monthly Notices of the Royal Astronomical Society, Volume 486, Issue 1, June 2019.

[11] Julien Chabé, Eric Aristidi, Aziz Ziad, et al, "PML: a generalized monitor of atmospheric turbulence profile with high vertical resolution," Appl. Opt. 59, 7574-7584 (2020)

[12] Dimitar R. Kolev and Morio Toyoshima, "Satellite-to-ground optical communications using small optical transponder (SOTA) - received-power fluctuations," Opt. Express 25, 28319-28329 (2017). 
[13] Florian Moll, Dimitar Kolev, Matthew Abrahamson, Christopher Schmidt, Ramon Mata Calvo, Christian Fuchs, "LEO-ground scintillation measurements with the optical ground station Oberpfaffenhofen and SOTA/OPALS space terminals," Proc. SPIE 9991, Advanced Free-Space Optical Communication Techniques and Applications II, 999102,21 October, (2016).

[14] C. Fuchs, F. Moll, D. Giggenbach, C. Schmidt, J. Keim and S. Gaisser, "OSIRISv1 on Flying Laptop: Measurement Results and Outlook," 2019 IEEE International Conference on Space Optical Systems and Applications (ICSOS), Portland, OR, USA, pp. 1-5, (2019). 